\title{
P02.193. Yoga of awareness: a randomized trial in fibromyalgia: post intervention and 3 month follow up results
}

\author{
C Wright ${ }^{*}$, J Carson, K Carson, R Bennett, S Mist, K Jones \\ From International Research Congress on Integrative Medicine and Health 2012 \\ Portland, Oregon, USA. 15-18 May 2012
}

\section{Purpose}

Comprehensive fibromyalgia (FM) treatment requires medications, exercise and improvement of coping skills. However, existing exercise protocols exert inadequate analgesic effects and suffer from poor adherence. The central hypothesis of the study is that yoga practiced with concurrent substantive mindfulness will reduce pain-related fear, increase pain acceptance and pressure pain thresholds, resulting in long-term adherence.

\section{Methods}

Fifty-three women with FM were randomized to an 8 week RCT of 2 hours weekly supervised group yoga + mindfulness or wait listed control. Yoga + mindfulness consisted of gentle poses, meditation, breathing exercises, yoga-based coping instructions, and group discussions drawing strongly on the Kripalu school of yoga.

\section{Results}

Immediately post-intervention women assigned to the yoga + mindfulness program compared to wait listed controls showed significantly greater improvements on standardized measures of FM symptoms and functioning (Revised Fibromyalgia Impact Questionnaire, FIQR), including pain, pain pressure thresholds, fatigue, mood, pain catastrophizing, acceptance, and other coping strategies. Post-treatment results in the wait-list group largely mirrored results seen at post-treatment in the immediate treatment group, with the FIQR Total Score improving 31.9\% across the two groups. Follow-up results showed that patients sustained most of their post-treatment gains, with the FIQR total score remaining $21.9 \%$ improved at 3 months. Multilevel random effects models demonstrated that those who

Oregon Health \& Science University, Portland, Oregon, USA practiced more had greater improvements. Home practice was 31 minutes daily vs 40 minutes daily in the wait listed vs immediately treated groups, respectively.

\section{Conclusion}

This novel pilot RCT indicated that yoga + mindfulness may be a safe, effective and durable intervention for women with FM.

Published: 12 June 2012

doi:10.1186/1472-6882-12-S1-P249

Cite this article as: Wright et al:: P02.193. Yoga of awareness: a

randomized trial in fibromyalgia: post intervention and 3 month follow up results. BMC Complementary and Alternative Medicine 2012 12(Suppl 1): P249.
Submit your next manuscript to BioMed Central and take full advantage of:

- Convenient online submission

- Thorough peer review

- No space constraints or color figure charges

- Immediate publication on acceptance

- Inclusion in PubMed, CAS, Scopus and Google Scholar

- Research which is freely available for redistribution

Submit your manuscript at www.biomedcentral.com/submit
C Biomed Central 